\title{
Littérature française moderne et contemporaine : histoire, critique, théorie
}

\section{Antoine Compagnon}

\section{(2) OpenEdition}

1 Journals

Édition électronique

URL : https://journals.openedition.org/annuaire-cdf/15626

DOI : $10.4000 /$ annuaire-cdf.15626

ISBN : 978-2-7226-0572-5

ISSN : 2109-9227

Éditeur

Collège de France

Édition imprimée

Date de publication : 30 décembre 2020

Pagination : 415-436

ISBN : 978-2-7226-0516-9

ISSN : 0069-5580

\section{Référence électronique}

Antoine Compagnon, «Littérature française moderne et contemporaine : histoire, critique, théorie »,

L'annuaire du Collège de France [En ligne], 118 | 2020, mis en ligne le 01 avril 2021, consulté le 22 août 2022. URL : http://journals.openedition.org/annuaire-cdf/15626 ; DOI : https://doi.org/10.4000/

annuaire-cdf. 15626 


\title{
LITTÉRATURE FRANÇAISE MODERNE ET CONTEMPORAINE : HISTOIRE, CRITIQUE, THÉORIE
}

\author{
Antoine COMPAGNON
}

Professeur au Collège de France

\begin{abstract}
Mots-clés : littérature, compétition littéraire, politique, pamphlet, duel, censure, Paul-Louis Courier, Armand Carrel, Granier de Cassagnac, Sainte-Beuve, Louis Veuillot

La série de cours et séminaires « De la littérature comme sport de combat (suite) » est disponible, en audio et vidéo, sur le site internet du Collège de France (https://www. college-de-france.fr/site/antoine-compagnon/course-2017-2018.htm), ainsi que les colloques « Histoire littéraire : nouveaux objets, nouvelles méthodes » (https://www. college-de-france.fr/site/antoine-compagnon/symposium-2017-2018.htm) et "Einstein au Collège de France » (https://www.college-de-france.fr/site/antoine-compagnon/ symposium-2017-2018__.
\end{abstract}

\section{ENSEIGNEMENT}

COURS ET SÉMINAIRES - DE LA LITTÉRATURE COMME SPORT DE COMBAT (SUITE)

Le cours de l'année dernière s'est intéressé aux tropes de la guerre littéraire des années 1820-1870, en recensant les figures selon un ABC (athlète, bravo, condottiere) resté incomplet, mais qu'avaient clos deux figures de réconciliation : celle du repos du guerrier et celle de la paix des loyaux adversaires. Il restait à examiner toutes les figures intermédiaires de cet alphabet. La solution proposée pour cette année sera celle que Les Antimodernes en leur temps avaient déjà éprouvée : à la liste des figures succédera un palmarès des grands soldats de cette guerre littéraire, qui éclaireront indirectement en retour les tropes demeurés inexplorés.

\section{Séance 1 - Introduction. Paul-Louis Courier, l'homme du pamphlet (I)}

9 janvier 2018

Paul-Louis Courier, ancien officier devenu journaliste, serait au premier rang de ces combattants littéraires : il invente le pamphlet moderne et meurt assassiné. Après 
lui viendraient Armand Carrel, lui-même lieutenant avant de devenir journaliste, mort lors d'un duel avec Émile de Girardin en 1836, puis Henri de Latouche, auteur de Fragoletta, connu pour son fiel, puis Béranger, le chansonnier républicain, Lamennais et Montalembert, militants catholiques, Louis de Cormenin, etc. Barbey d'Aurevilly pourrait constituer le terme de la liste, à moins que, décidant d'y intégrer Chateaubriand au départ, on ne se retrouve tenté de la compléter de l'autre côté jusqu'à Léon Bloy.

Ces combattants peuvent souvent être disposés par couples : à Latouche et sa « camaraderie littéraire » répond Gustave Planche et sa « haine littéraire »; Nisard et Janin s'opposent sur la question de la littérature facile ; si Granier de Cassagnac attaque en "ligne droite », Janin préfère la «ligne courbe »; Veuillot s'oppose à Granier de Cassagnac, comme le sacré et le profane, mais il s'oppose aussi à Proudhon comme une violence de droite se distingue d'une violence de gauche.

$\mathrm{Au}$ centre de ce réseau, on ne cesse de retrouver Sainte-Beuve, qui connaît tous ces combattants, a écrit des articles importants sur tous. La relation de Sainte-Beuve et Balzac, plutôt que celle de Sainte-Beuve et Hugo, est un modèle de guerre littéraire. Du reste, comme il l'avoue lui-même, tous les écrits du critique s'avèrent placés sous le signe de la revanche.

Paul-Louis Courier (1772-1825) fut un grand écrivain de la III ${ }^{\mathrm{e}}$ République, mort avec elle et avec la fin de l'anticléricalisme dont il s'était fait le champion. Il revint à Janin de le décrire comme un pamphlétaire : mais le mot, alors, prenant le sens qu'on lui connaît aujourd'hui, est un néologisme que Courier lui-même emploie peu. Il est officier d'artillerie de la Révolution et de l'Empire, en même temps que savant helléniste. Sa carrière militaire est peu brillante en raison de son indiscipline. Il se décrit comme canonnier et vigneron, très attaché à son identité de propriétaire terrien de l'Indre-et-Loire. Ayant quitté l'armée en 1809, il la rejoint à nouveau pour Wagram, puis déserte durant la bataille. Il réside en Italie jusqu'à la fin de l'Empire, revient à Paris en 1824, épouse la fille d'Étienne Clavier, qui avait été professeur de grec au Collège de France. En 1816, il écrit une Pétition aux deux chambres pour prendre la défense d'un paysan qui a refusé d'enlever son chapeau devant un prêtre : c'est le début de sa carrière contre la réaction royaliste, contre l'alliance du Trône et de l'Autel. Trois ans plus tard, il écrit un premier pamphlet contre l'Académie des inscriptions et belles-lettres, pour se venger de n'y avoir pas été élu à la succession de son beau-père. En 1821, il est l'auteur d'un Simple discours de Paul-Louis vigneron, écrit pour protester contre la souscription nationale lancée au profit du petit duc de Bordeaux pour l'acquisition du château de Chambord. Son procès est célèbre, il devient l'un des premiers condamnés aux termes de la loi de diffamation de 1819. Envoyé à SaintePélagie, il en profite pour rédiger son procès. Sa grande œuvre est son Pamphlet des pamphlets de 1824, par lequel il se réapproprie le terme de pamphlet qui avait été d'abord utilisé contre lui. Albert Thibaudet lui assigne une place essentielle dans son Histoire de la littérature française de 1936, comme représentant d'une tradition pamphlétaire de gauche avec Béranger et Stendhal, avant que le pamphlet ne vire à droite.

\section{Séminaire lié - Du journalisme à la littérature}

Intervenant : Emmanuel Carrère, journaliste et écrivain. 


\section{Séance 2 - Paul-Louis Courier, l'homme du pamphlet (II)}

16 janvier 2018

On retrouve souvent l'éloge de Courier sous la plume de Stendhal. Ce dernier, de dix ans son cadet, a traversé les guerres avec le même dilettantisme et déteste autant que lui l'ordre moral et le pouvoir temporel du clergé. Dans ses Chroniques, Stendhal fait de lui tantôt le Voltaire du XIX ${ }^{\mathrm{e}}$ siècle contrant les " autorités constituées », tantôt le donneur de nouvelles Provinciales, mort avant de devenir un second Pascal. L'auteur de La Chartreuse de Parme est un grand admirateur de sa Pétition pour des villageois que l'on empêche de danser (1822), du Simple discours sur la souscription de Chambord (1821); mais c'est la Pièce diplomatique de 1823 qu'il préfère, texte que Courier attribue à Louis XVIII, écrivant à son frère Ferdinand d'Espagne. Le roi y fait un éloge inattendu du système représentatif, détourné en «gouvernement récréatif » dans lequel il s'amuse à nommer lui-même les députés du peuple. Stendhal se met à écrire des pamphlets à la suite de Courier. Thibaudet perçoit en 1936 la veine romanesque des pamphlets anticléricaux de Courier et suggère que les personnages du Rouge et le Noir, voire les curés de Balzac, héritent beaucoup du clergé de Luynes sur qui Courier fait porter sa satire.

Courier, dont la vie est brève, doit sa renommée à une tache d'encre. Lors de la campagne d'Italie, il découvre dans une bibliothèque de Florence un manuscrit du Daphnis et Chloé de Longus, où figure un passage absent de toutes les versions connues, et notamment de la traduction d'Amyot datant de la Renaissance. En 1809, Courier retourne à Florence pour transcrire les six ou sept pages capitales où est décrite la naissance du sentiment amoureux ; au moment de transcrire, il fait « un pâté sur le Longus » qui, obscurcissant le précieux passage, devient une affaire d'État. On l'accuse d'avoir agi délibérément pour garantir l'exclusivité de sa découverte.

Son assassinat, le 10 avril 1825, dans le bois de Larçay dont il est propriétaire, achève d'établir son mythe. La rumeur court longtemps d'un crime politique ourdi par le parti jésuite, quoique l'hypothèse d'un règlement de compte familial ait été proposée très tôt. En 1829, il apparaît que la mort de Courier a été orchestrée par deux de ses anciens domestiques avec lesquels sa femme le trompait. La mort de Courier parfait son image de pamphlétaire misanthrope mais surtout dilettante, solitaire, entièrement coupé de la camaraderie littéraire.

Dans son Pamphlet des pamphlets de 1824, il se met en scène prenant la route de Sainte-Pélagie, et rencontrant un des jurés de son procès, qui lui apprend ce que signifie ce mot de pamphlétaire sur le chef duquel on l'incrimine. Toute la question est celle d'un glissement sémantique : le pamphlet désigne d'abord en termes neutres un court prospectus. Cette brièveté suscite la méfiance car elle est la garantie que Courier est davantage lu que les historiens aux lourds ouvrages. Le danger du format court explique l'emploi de plus en plus métaphorique du terme pamphlet. Pour sa défense, Courier rappelle à son interlocuteur fictif que les Provinciales ne sont rien d'autre que de très courtes lettres, qui figurent néanmoins au canon des œuvres classiques.

\section{Séminaire lié - Lignes du front}

Intervenante : Florence Aubenas, grand reporter au Monde. 


\section{Séance 3 - Paul-Louis Courier, I'homme du pamphlet (III)}

\section{3 janvier 2018}

Les pamphlets de Courier, vendus « chez tous les marchands de nouveautés »- les nouveautés sont les brochures éphémères, mais non périodiques - rencontrent un franc succès, ce que Balzac évoque notamment dans Illusions perdues. Le romancier souligne aussi que le pamphlet est toujours un texte d'opposition au pouvoir. Énumérant les noms des grands pamphlétaires du siècle - Constant, Chateaubriand, Courier, Vatout, plus récemment Lamennais et Cormenin, ou Béranger - l'auteur de La Comédie humaine accorde à Courier de rejoindre le canon : sa cible a peut-être disparu avec la Restauration, mais son monument littéraire demeure.

Cormenin est une figure intéressante : il est député d'opposition pendant toute la monarchie de Juillet, vote systématiquement contre le gouvernement mais ne prend jamais la parole à la Chambre. Il semble qu'il fasse de son silence le moyen d'une surveillance scrupuleuse du régime, mais aussi de la naissance de l'éloquence parlementaire. Il publie en 1842 un Livre des orateurs, où il expose à partir de l'exemple de ses collègues une théorie de la rhétorique parlementaire, journalistique et pamphlétaire. Il conçoit l'auteur de pamphlet comme le «soldat de la presse militante ». On blâme néanmoins Cormenin pour la lourdeur, l'incohérence de son style. Balzac qui définit le pamphlet comme «le sarcasme à l'état de boulet de canon » lui reproche d'avoir le style « filandreux » plutôt que « l'allure à la Figaro de Courier».

La guerre de Courier est en grande partie politique, mais elle est aussi littéraire : il déteste tous les écrivains depuis le XVII ${ }^{\mathrm{e}}$ siècle, raillant «les Jean-Jacques, Diderot, d'Alembert [...] sous le rapport de la langue ». À son éditeur qui l'appelle helléniste, il répond qu'helléniste rime avec dentiste, droguiste, ébéniste : on veut le faire savant de métier ou par politique, faiseur de livres comme Chateaubriand, mais lui revendique le loisir studieux de Montaigne.

C'est toujours sur une erreur de langue, sur un détail d'expression, plutôt que sur la thèse adverse, que Courier fait porter son attaque, pour ainsi dire en philologue minutieux. Il raille Chateaubriand dans son grand discours à la Chambre, au retour du Congrès de Vérone. À Lamartine il reproche ses phrases «hors de ce commun langage que tout le monde parle et entend ». Le « commun langage » c'est la langue du frais XVI ${ }^{\mathrm{e}}$ siècle, contre les raffinements spécieux de la cour de Louis XIV. C'est la langue de Rabelais, de Montaigne ou d'Amyot - Courier a imité son style pour traduire les passages manquant de Daphnis et Chloé - et c'est encore la langue des paysans. Cette haine pour l'académisme néoclassique le rapproche encore de Stendhal, et explique que Delescluze ait pu ranger les textes de Courier, avec la préface de Cromwell et le Racine et Shakespeare de Stendhal, parmi les influences de la bataille romantique. Stendhal lui-même se reconnaît dans cet intérêt qu'a Courier pour le mot propre, contre la rage d'ennoblir du siècle précédent.

Quant à Courier et Chateaubriand, ils s'estiment, en dépit des critiques répétées du premier et de leur opposition politique. Chateaubriand consacre une longue digression à Courier dans la Vie de Rancé et rend même un hommage ému au pamphlétaire assassiné, évoquant à son propos le chant d'une grive rappelant celle de Montboissier. Courier avait donné l'hommage réciproque dans une lettre de 1824, peu de temps avant sa mort reconnaissant en Chateaubriand l' « écrivain véritable [...] poète, comme nul en prose ne le fut jamais », malgré son goût pour Rousseau, Bernardin ou Shakespeare. Courier est dans toutes les anthologies scolaires de la 
III République, mais il disparaît avec elle : la dernière édition d'ampleur est celle de la Pléiade de 1940, juste avant la guerre et le début d'un régime qu'il eût vilipendé.

\section{Séminaire lié - Regard médical, regard littéraire : deux processus complémentaires?}

Intervenant : Jean-Christophe Rufin, médecin, écrivain, membre de l'Académie française.

\section{Séance 4 - Armand Carrel, sous-lieutenant et journaliste}

\section{0 janvier 2018}

Armand Carrel est unanimement reconnu comme le digne successeur de Courier. Ils sont tous deux officiers, autodidactes, quittent tous deux l'armée pour la plume, conservent tous deux une sorte de discipline de l'indiscipline; ils comptent tous deux parmi les saints patrons de la III ${ }^{\mathrm{e}}$ République.

Carrel, qui naît en 1800, est contemporain de la grande génération romantique ; sa mort dans le duel qui l'oppose à Émile de Girardin, patron de La Presse, journal adversaire du National, dirigé par Carrel, contribue à asseoir son mythe. Admiré de ses collaborateurs comme de ses adversaires, c'est un « soldat-poète », un Byron français. Littré, son premier biographe, dit que toute sa vie s'inscrit entre ces deux appellations, le sous-lieutenant et le journaliste. Chateaubriand, reprochant à la Restauration de n'avoir su donner un rôle à Carrel, le trouve militaire dans sa pratique de journaliste, puisque journaliste à défaut d'avoir pu devenir le grand militaire qu'il devait être.

Comme Vigny, Carrel est un soldat venu trop tard. Élevé dans les lycées de l'Empire, dans l'admiration de la Grande Armée, il entre à Saint-Cyr en 1811, malpensant et indiscipliné. À Verdun où il se trouve bientôt sous-lieutenant dans un régiment d'infanterie, il fréquente les cabinets de lecture et lit les «mauvais journaux », prend des premiers contacts avec la charbonnerie, les sociétés secrètes. Il commence une relation durable avec Émilie Antoine, la femme de son capitaine, avec qui il se bat en duel en 1823. Sa vie de jeune homme rappelle celle de Lucien Leuwen, inventé par Stendhal au moment de la grande gloire de Carrel. Les années 1821-1824 sont marquées par les conspirations militaires, dont l'affaire retentissante des quatre sergents de La Rochelle, guillotinés en place de Grève pour avoir fomenté un coup contre la monarchie. Carrel lui-même est mêlé à une conspiration, à Belfort, mais n'est pas repéré. Son bataillon est envoyé à Marseille pour mise au pas.

Carrel est réformé le 18 mars 1823, juste avant que son bataillon ne soit envoyé en Espagne en soutien à la monarchie carliste, conformément au souhait de Chateaubriand ministre. Embarqué sur un bateau de pêche, il rejoint le camp des constitutionnels espagnols, au sein de la Légion étrangère. Il combat, sous le drapeau tricolore et l'aigle napoléonien, contre ses compatriotes de l'armée de Restauration.

Chateaubriand, paradoxalement, admire Carrel. Dès avant de le connaître, il cite dans la préface de ses Études historiques l'article que Carrel a écrit en 1828 sur la guerre d'Espagne. Amenés à se rencontrer, les deux hommes deviennent amis. Chateaubriand se reconnaît en lui : Carrel est lui aussi à sa manière l'homme d'une cause perdue d'avance, l'homme de la fidélité à un passé.

En septembre 1823, à Figueras, c'est à Carrel qu'il revient de négocier la capitulation de la Légion étrangère avec le général de Damas, qui promet de demander sa grâce. Carrel est néanmoins jugé par un conseil de guerre qui se déclare 
incompétent, puisqu'il a quitté les rangs de l'armée française avant de se rendre en Espagne. Le jugement est révisé une première fois, et Carrel condamné à mort, puis acquitté par une nouvelle révision. Entre-temps, il profite de son incarcération pour lire et faire ses premières armes contre la Restauration.

Il devient secrétaire de l'historien Augustin Thierry, achève le manuscrit de son Histoire de la conquête d'Angleterre par les Normands. En 1827 surtout, Carrel publie l'Histoire de la contre-révolution en Angleterre sous Charles II et Jacques II, son premier livre et véritable pamphlet contre la Restauration, sous l'apparence d'un livre d'histoire. Carrel veut délégitimer Charles X, substituer ce qu'il appelle la royauté consentie, c'est-à-dire la lignée d'Orléans, à la royauté légitime. Il s'affirme comme le partisan d'une ligne libérale, contre la référence au droit divin. Toutefois, Carrel n'est pas révolutionnaire : en 1830, il reste en retrait des événements, quoiqu'il les salue après coup. Il est défenseur de la Charte plutôt que de la souveraineté du peuple.

Thiers, Mignet et Carrel fondent le National en 1830, mais après les événements de Juillet, Carrel en devient le seul directeur.

\section{Séminaire lié - Du brouillard de la guerre au brouillard de la paix : la guerre au $X X l^{e}$ siècle}

Intervenant : Jean-Marie Guéhenno, diplomate.

\section{Séance 5 - Armand Carrel, l'esprit de chevalerie}

\section{6 février 2018}

Après les Trois Glorieuses, Armand Carrel fait amende honorable et admet ne pas avoir compris les événements. Demeuré seul directeur du National - Thiers et Mignet ont été appelés à d'autres fonctions -, Carrel clarifie très vite sa ligne : il importe plus que jamais que le National existe, puisque c'est sa ligne qui vient tout d'un coup de s'imposer, mais son nouveau directeur se gardera bien de le laisser devenir une feuille ministérielle. Or, le journaliste, avec ses articles, est bien plus redoutable que tout autre opposant au pouvoir en place, ce dont se souviendra Stendhal dans les dialogues de Lucien Leuwen.

Le lien d'identification est fort entre le National et Carrel, qui menace de duel tous les adversaires politiques de celui-là. Carrel déploie dans ses pages tout son élan de militaire : au service de la liberté de la presse ; contre la liste civile ; contre CasimirPerier, président du Conseil à partir de mars 1831 ; contre le projet de construire un fort à Montmartre ; surtout contre l'hérédité à la Chambre des pairs, dont l'abolition en octobre 1831 est sa grande victoire.

Début 1832, Carrel se sépare définitivement du régime et se déclare républicain, avec en tête désormais le modèle américain plutôt que l'anglais. Mais il se fait une idée autoritaire de la République et ce libéral, se rêvant "préfet de police vingtquatre heures pour mettre tout ce monde à la raison », paraît quelque peu paradoxal. Son ethos militaire le prive d'une intuition des révolutions en cours.

Ses relations avec les autres républicains sont conflictuelles. La Tribune lui reproche son ton condescendant et professoral ; il est la première incarnation d'une aristocratie intellectuelle. Carrel est favorable à la décentralisation, comme les légitimistes, et contre les républicains qui sont jacobins. Détracteur d'un républicanisme de sentiment, il prône un républicanisme de raison, choisi par ceux que la force des choses et la succession des régimes ont fait républicains. Au moment 
où l'on dénonce les girouettes, Carrel fait leur éloge : ce sont gens à avoir mis le sens de l'État au-dessus de la notion de régime. En prison à Sainte-Pélagie, Carrel refuse de commémorer le 21 janvier, date de la mort de Louis XVI : il s'attire les foudres de ses camarades républicains.

Littré et Nisard sont pour beaucoup dans la transformation de Carrel en saint républicain, volontaire et loyal. Il est le héros de la moralité républicaine, l'étalon moral de la presse d'opposition. Ainsi, il écrit un article célèbre pour défendre le chef basque espagnol Zumalacarregui, dont il ne partage pourtant pas les idées. Il nourrit de même une amitié pour Chateaubriand dont il annonce les pamphlets dans les colonnes du National. Carrel signale son procès après son Mémoire sur la captivité de $M^{m e}$ la duchesse de Berry et prend sa défense au nom de la liberté de la presse; il l'accueille à la sortie du tribunal qui l'acquitte. Il reproduit dans son journal les premiers extraits à paraître des Mémoires d'outre-tombe.

À partir de 1832, le National fait l'objet de procédures judiciaires toujours plus nombreuses, menées par le procureur Persil. Les procès pour délits de presse sont instruits par les cours d'assises : Carrel assure lui-même sa défense devant les jurys, obtient toujours son acquittement. En janvier 1832, le ministère de Casimir-Perier restaure l'arrestation préventive pour les délits de presse : Carrel appelle explicitement à la résistance et remporte le procès qui lui est fait à cette occasion, ainsi qu'un autre à la suite des insurrections de juin 1832. Le ministère change son angle d'attaque : on utilise une subtilité de la loi de 1822 qui punit l'infidélité et la mauvaise foi des journaux qui rendent compte des audiences des Chambres et des tribunaux. Pour ces crimes-là, les Chambres sont autorisées à se constituer seuls juges en même temps que parties, sans jury. C'est une tentative explicite pour museler la presse d'opposition. Après l'affaire du coup de pistolet intenté contre Louis-Philippe, le National se voit interdit de rendre compte des procès pendant deux ans, ce qui risque de nuire à son équilibre économique. Carrel fait appel aussi longtemps qu'il le peut, transforme le National en National de 1834 pour retarder l'échéance, le temps qu'on juge s'il s'agit ou non du même journal. Le 30 août 1834, le feuilleton prend fin : Carrel perd, est condamné à six mois d'emprisonnement à Sainte-Pélagie.

\section{Séminaire lié - Plier, déplier : mémoires au présent}

Intervenant : Laurent Mauvignier, écrivain.

\section{Séance 6 - Carrel, la salutaire prévoyance du duel}

\section{3 février 2018}

Carrel passe les mois d'octobre 1834 à avril 1835 à Sainte-Pélagie : c'est alors qu'il connait le moment le plus glorieux de sa carrière. La Chambre des pairs, constituée en Haute Cour, a entrepris de juger 184 responsables des événements d'avril 1834. Dans le National du 10 décembre, Carrel fait paraître sous anonymat un article virulent qui remet en cause la légitimité de la Cour des pairs, vestige de la Restauration, pour instruire un tel procès. Le lendemain, le comte Philippe de Ségur invoque pour la première fois l'article 15 de la loi du 25 mars 1822, qui permet aux Chambres de juger directement ceux qui les offensent. On décide de traduire Rouen, le gérant du National. Le 12 décembre, Rouen comparaît devant la Chambre mais demande à être assisté de Carrel. Le 16, Carrel se présente. Dupin aîné, président de la Chambre des députés, est présent, ainsi que le Lord Chancelier Brougham, ancien président de la Chambre des 
pairs britannique. L'audition de Carrel a lieu trois jours après le discours de réception à l'Académie française de Thiers, son ancien ami, cofondateur du National, entre-temps devenu ministre de l'Intérieur et responsable de la répression des événements d'avril, notamment lors de l'épisode de la rue Transnonain.

Carrel raille la Chambre, cite d'anciens articles que Thiers lui-même avait écrits contre elle. Il ne manque pas de se référer à Lord Brougham, qui a montré dans son propre pays l'exemple d'un comportement libéral envers un journaliste qui l'avait offensé. Devant le baron Pasquier il fait allusion au maréchal Ney, condamné, selon lui, par une Chambre indigne, ce en quoi l'approuve le général Exelmans lui-même. Rouen est déclaré coupable et condamné à deux ans de prison et dix mille francs d'amende, presque la peine maximale. Dès le lendemain, le National lance une souscription nationale sous l'intitulé «Protestation contre l'assassinat du maréchal Ney ». On dit que le duc d'Orléans lui-même y a contribué en secret.

Le 28 juillet 1835, Fieschi lance son attentat à la «machine infernale»contre Louis-Philippe, et fait 19 morts. La répression est virulente, et Carrel est arrêté dès le lendemain matin, accusé d'avoir excité les conspirateurs à l'action. Les lois de septembre 1835 sont en préparation : il s'agit d'en finir avec la liberté de la presse. On établit le cautionnement des journaux, on interdit la souscription pour payer les amendes, les caricatures sont soumises à censure préalable. C'est cette loi qui prépare le remplacement des journaux d'opinion par une nouvelle presse, dont $L a$ Presse est représentative. À Sainte-Pélagie, Carrel bénéficie d'un traitement de faveur : il continue de publier, a reçu l'autorisation de sortir quand il le veut. Écrivant à Chateaubriand, il se dit le « jeun[e] solda[t] d'une cause dont [il est] le vétéran le plus glorieux»: c'est la liberté de la presse. Chateaubriand lui répond: «la République est la plus belle de vos chimères ».

\section{Séminaire lié - Une grammaire du massacre}

Intervenant: Jacques Semelin, historien, professeur à Sciences Po et directeur de recherches au CNRS, spécialiste des violences de masse et des phénomènes de résistance.

\section{Séance 7 - Carrel et « ce bois fatal [qui] m'a fait pleurer deux fois »}

\section{0 février 2018}

Armand Carrel est mêlé au duel qui oppose le député François-Charles Dulong au général Bugeaud, le 29 janvier 1834. Dulong a offensé ce dernier lors d'un débat sur l'autorité militaire. Les deux hommes s'affrontent et Dulong meurt. Bugeaud accuse Carrel, ami de Dulong, d'avoir causé sa mort en le dissuadant de publier ses excuses. Carrel, lui, se persuade que c'est Louis-Philippe qui est commanditaire d'un meurtre politique. Le témoin de Bugeaud, en effet, n'est autre que le général de Rumigny, aide de camp de Louis-Philippe, que Carrel accuse d'avoir empêché la réconciliation. Rumigny tient le cabinet noir, la police parallèle de Louis-Philippe ; c'est lui qui recrute les casseurs lors des manifestations républicaines, pour justifier un plus dur écrasement de la contestation.

Après cette histoire, Dupin aîné essaye une première fois d'interdire le duel ; Carrel s'en fait contre lui l'apologiste. Il veut maintenir le duel comme seule protection contre la calomnie, parce que grâce à lui la liberté d'expression se trouve garantie. En 1837, juste après la mort de Carrel par voie de duel, Dupin aîné obtient 
de la Cour de cassation que cette pratique, à défaut d'être interdite, soit remise dans le droit commun, les duellistes pouvant être poursuivis pour homicide.

Est-ce l'habitude militaire, que Carrel conserve dans son métier de journaliste, qui le condamne à reprendre si souvent l'épée ? Il se bat trois fois en duel en tant que directeur du National : la première, au tout début de l'existence du journal, contre le directeur du Drapeau blanc, puis la deuxième fois le 2 février 1833. La rumeur de la grossesse de la duchesse de Berry vient d'arriver à Paris, et provoque une épidémie de duels entre républicains et carlistes. De nombreux journaux républicains sont pris à partie par le camp carliste : Carrel y voit une tentative de censure, et répond en proposant le duel. Mais le préfet Gisquet a entrepris d'arrêter tous les fauteurs de trouble potentiels : Carrel voit là encore et surtout un risque de censure de la presse, au-delà de la différence des opinions.

Carrel semble prêt à faire amende honorable et à quitter l'arme pour la plume. Toutefois, le 22 juillet 1836, il affronte Émile de Girardin dans le duel qui le condamne. C'est le conflit de la vieille presse politique et de la nouvelle presse, juste après les lois de septembre 1835 qui rétablissent un cautionnement élevé et la censure préalable pour les caricatures. Girardin, juste après le lancement de son journal, a publié un article hostile à la presse politique ; celle-là lui répond à travers un article du Bon Sens, que Girardin en retour décide d'attaquer pour diffamation devant le tribunal correctionnel. Carrel s'irrite du procédé : Girardin a fait appel à la justice corrompue, incompétente selon Carrel, plutôt que de choisir la voie de l'honneur. Girardin réplique une dernière fois en menaçant de révéler dans ses colonnes la vie intime du directeur du National, lequel vit avec la femme de son ancien capitaine.

Carrel meurt des suites d'un coup de pistolet le 24 juillet au matin. Chateaubriand lui rend un hommage émouvant dans ses Mémoires, évoquant à son propos "l'ombre du petit-fils du grand Condé ». C'est selon une figure analogue que Chateaubriand avait comparé la mort de Courier à celle de Rancé. On retrouve enfin Carrel dans le Congrès de Vérone, en 1838 où Chateaubriand donne la première ébauche de ce qui deviendra le célèbre appel aux morts des Mémoires d'outre-tombe.

\section{Séance 8 - Granier de Cassagnac, plume de guerre}

\section{0 février 2018}

Adolphe Granier de Cassagnac, né en 1806, est à peine plus jeune que Carrel ; il est le plus violent des guerriers littéraires de l'époque. Baudelaire l'oppose à Janin comme rare représentant de l'attaque en « ligne droite » dans les pamphlets. Granier est le modèle du condottiere : véritable mercenaire qui, dit Veuillot, ne cesse de passer d'un camp à l'autre. Barbey prétend que Granier se sauve en n'ayant jamais offert ses services aux partis du mouvement, où sa participation aurait été bien plus rentable ; Larousse fait remarquer que Granier dans sa jeunesse a néanmoins signé une brochure démocrate. Granier quitte Toulouse pour Paris en 1832, accompagné d'une lettre de recommandation auprès du député de la Haute-Garonne Charles de Rémusat. Grâce à lui, il rencontre très vite Guizot, Hugo puis Bertin, le directeur du Journal des Débats. Il y met immédiatement ses talents de polémiste au service de Hugo, et acquiert sa renommée par un seul article qu'il écrit contre Dumas, pour dénoncer ses plagiats. Granier de Cassagnac n'est pas de ceux qui ont quitté l'épée pour la plume, mais sa plume est d'emblée une épée. Granier relève tous les plagiats 
de Dumas. Dans un article, il dispose en deux colonnes la comparaison des textes de Dumas avec ceux d'Augustin Thierry et de Chateaubriand. Hugo fait bien évidemment partie des plagiés. Le critique est bientôt obligé de révéler son nom, car on accuse Hugo d'avoir téléguidé sa plume. Granier revendique l'initiative de son article et dit qu'Hugo a plutôt essayé de le retenir. Dumas répond par sa théorie de la conquête, qu'il formule dans son article «Comment je devins auteur dramatique ». Granier y voit l'aveu de sa victoire.

Quelque temps plus tard, Granier entreprend d'exécuter Racine dans les colonnes de La Presse. On est en pleine bataille romantique, et Granier semble, ce faisant, s'approprier une réflexion de Heine, qui note, en fin observateur de la vie démocratique, l'absurdité de certains débats. Granier consacre dix articles à Racine tout au long de 1838, lesquels assurent son statut de célébrité parisienne. En 1839, il polémique avec Janin, qui se demande si Victor Hugo sait bien le français et déplore les barbarismes du grand romantique. Granier lui répond que savoir une langue, c'est non pas connaître le dictionnaire mais les grands auteurs qui l'ont faite. Par la suite, Granier est embrigadé dans le parti colonialiste, il écrit des brochures contre l'affranchissement des esclaves. Il part aux Antilles d'octobre 1840 à juin 1842, épouse Rosa de Beaupin de Beauvallon, fille d'un planteur de Guadeloupe. De retour, il écrit au Globe, le quotidien du parti esclavagiste. Sa dernière grande polémique est à l'occasion du duel de son beau-frère, Rosemond de Beauvallon, qui tient le feuilleton théâtral du Globe, contre Dujarrier, acolyte de Girardin à La Presse. Dujarrier meurt au cours du duel, le 11 mars 1845, mais Rosemond de Beauvallon est poursuivi pour assassinat avec préméditation, parce qu'il apparaît qu'on s'est servi des pistolets de Granier de Cassagnac, avec lesquels il a pu avoir le temps de se familiariser avant le duel. La cour d'assises de Rouen finit néanmoins par l'acquitter. Son avocat a cité Guizot, défenseur du duel qui y voit lui aussi la garantie de la liberté de la presse.

\section{Séance 9 - Ce diable de Veuillot}

6 mars 2018

Trois raisons au moins donnent lieu de s'intéresser à Louis Veuillot. D'abord, il prend une part importante, au milieu du siècle, dans le débat sur la place à accorder à la culture classique dans l'enseignement. En 1851, l'abbé Gaume publie Le Ver rongeur des sociétés modernes, ou le Paganisme dans l'éducation, qui condamne l'introduction des textes de l'Antiquité païenne, celle-là même qu'a ravivée la Renaissance, dans l'éducation des jeunes catholiques. Veuillot lui apporte son soutien dans L'Univers, qu'il dirige ; il s'oppose à Mgr Dupanloup, évêque d'Orléans, figure du légitimisme éclairé ; l'affaire va jusqu'à Rome et Pie IX doit répondre par l'encyclique Inter multiplices qui critique le gallicanisme et affiche finalement une position modérée sur la question des classiques.

Deuxième curiosité : Veuillot est fondateur d'un certain style pamphlétaire français, que Thibaudet reconnaît après lui dans Drumont, Bloy, Daudet, Maurras. Il tient en grande partie à la trajectoire sociale de Veuillot, autodidacte issu du peuple, qui finit par s'adresser, avec L'Univers, au petit peuple catholique de province. Hugo s'en prend à Veuillot après 1851 car ce dernier s'est fait défenseur du coup d'État : il le raille dans Châtiments, non sans lui reprendre la langue du peuple dont celui-ci s'était fait le relais. 
Veuillot est enfin l'ami paradoxal de Baudelaire (et de Nadar) : est-ce à cause de sa curiosité pour le mal, pour le péché ? Tous les deux sont également disciples de Joseph de Maistre. Cette amitié, sur fond d'alliance des contraires, n'est pas sans remous. Veuillot raille un article de la Société des gens de lettres sur la mort de Nerval, en 1855. On y dit que Nerval a «trop spiritualisé la vie »; Veuillot répond qu'il l'a probablement trop alcoolisée. Il parle en connaisseur, ayant lui-même appartenu à cette bohème poète et journaliste avant sa conversion en 1838. Outré par le passage, Baudelaire lui envoie néanmoins Les Fleurs du Mal; Veuillot en rend compte en 1858, considérant dans son article que l'auteur souffre de la comparaison avec Musset. Si le critique est présent dans le cortège funéraire de Baudelaire, il continue, par la suite, à réserver ses jugements sur les textes du poète.

Veuillot est incessamment caractérisé selon deux régimes contradictoires. Sa laideur physique dégoûte mais il amuse par sa méchanceté et sa grossièreté ; d'après un journaliste contemporain, son style, chargé de "pétarades horrifiques et épouvantables [...], pue le cabaret et la sacristie ». Sa carrière naissante rappelle celle des petits journalistes de la Restauration et de la monarchie de Juillet que Balzac décrit dans Illusions perdues. Ses parents sont illettrés, lui-même ne va pas au collège. Il débute en 1825, en pleine bataille romantique, chez l'avoué Fortuné Delavigne, passionné de littérature et frère du poète Casimir Delavigne. Il devient par la suite un véritable condottiere, journaliste à gages, écrivant systématiquement pour les journaux ministériels. À 19 ans, le général Bugeaud l'envoie à Périgueux pour y tenir le Mémorial de la Dordogne; Guizot, son autre patron, le rappelle à Paris en 1836 pour la rédaction de La Charte de 1830. Il entre ensuite au Moniteur parisien où il rend compte des débats parlementaires. Mais il s'effraie de perdre sa morale, voyage à Rome en 1838, se convertit et s'éloigne momentanément du milieu journalistique pour rejoindre l'Administration de l'esprit public (les futurs Renseignements généraux). En 1841, Bugeaud l'emmène avec lui en Algérie ; il y prend le sabre, contribue à la prise de Médéa et de Mascara, mais a la nostalgie de la bataille des plumes et des idées. En 1843, il quitte le ministère de l'Intérieur, devient rédacteur en chef de L'Univers catholique. Il adhère, ce faisant, aux idées de Joseph de Maistre, détestant avant Maurras la triade Renaissance, Réforme, Révolution. On ne cessera de rappeler l'origine populaire de Veuillot; il apparaît aux yeux de beaucoup comme l'introducteur de l'esprit démocrate dans l'Église, jusqu'ici dirigée par une aristocratie. Son premier combat, dans les années 1840, est pour la liberté de l'enseignement catholique, contre la loi que Villemain prépare sur les congrégations, qui doit bientôt donner le monopole de l'enseignement à l'Université. Il incarne le polémiste catholique intransigeant, resté libre jusqu'au bout, refusant aussi bien la Chambre que l'Académie.

\section{Séminaire lié - De la nécessité du reportage de guerre}

Intervenante : Anne Nivat, grand reporter.

\section{Séance 10 - Veuillot et la « vieille citrouille à moitié remplie de diamants »}

\section{3 mars 2018}

Veuillot, jeune romantique, avait fait partie de la claque d'Hernani. Dans L'Écho de Rouen, en 1831, il avait rendu compte des Feuilles d'automne, faisant l'éloge d'Hugo quoique regrettant déjà le virage politique du poète. En 1842, il 
consacre un feuilleton très sévère au Rhin, dénonce les hugolâtres, critique les facilités de plume du poète, et surtout sa prétention à guider le peuple. Il condamne l'évolution politique d'un Hugo d'abord royaliste, puis quasi chrétien sous l'influence de Chateaubriand, puis libéral à la façon des Débats, puis révolutionnaire. La rupture est consacrée en 1851. Si Veuillot est paradoxalement favorable aux événements de 1848 - selon le schéma très maistrien d'une Providence se manifestant dans le mouvement du peuple -, il rejoint bientôt le parti de l'ordre. En février 1851, Veuillot approuve le coup d'État, et son journal appelle à voter oui au plébiscite.

Sa décision lui vaut d'être l'une des figures les plus maltraitées de Châtiments encore que les deux pièces consacrées par Hugo à Veuillot, «À des journalistes de robe courte » et «Un autre », soient datées de septembre 1850, attestant une présence de Veuillot très tôt dans le projet du recueil. Hugo se venge en particulier des comptes rendus sévères que Veuillot donne de ses discours à l'Assemblée, qui dénoncent sans cesse la trahison de l'ancien pair de France devenu orateur de la France populaire, à la différence de Montalembert resté fidèle à ses origines.

Le 19 octobre 1849, on débat à l'Assemblée de la restauration des pouvoirs temporels du Saint-Siège; Victor Hugo prononce l'un de ses plus importants discours, qui marque son passage au radicalisme. Veuillot se moque des antithèses de Hugo, devenu poète essoufflé depuis qu'il a grimpé la Montagne. Dans ses railleries, le critique trouve un allié en la personne de Montalembert. Mais Hugo rétorque à propos de ce dernier : «il a passé du côté de ceux qui oppriment, et moi je reste du côté de ceux qui sont opprimés. »

Le débat sur le suffrage universel achève d'attiser leur guerre. Veuillot raille encore le discours de Hugo, homme politique et poète. Dans son livre sur les Libres penseurs, en 1860, il déclarera : "Le poète est un moineau lascif, c'est le fond de sa nature ». Mais la phrase disparaît de la réédition du livre: Veuillot, catholique, s'est-il rendu compte qu'il citait un vers érotique de Catulle ? Entre-temps, Émile Deschanel lui répond que « si le poète est un moineau lascif, le pamphlétaire clérical est un vilain hibou ». Les moqueries de Veuillot n'épargnent pas non plus le second grand discours de Hugo à l'Assemblée, lors de la révision de la Constitution en 1851.

En dépit de désaccords majeurs, Veuillot influence Hugo dans l'écriture de plusieurs pièces de Châtiments : il «encanaille la muse de l'ancien pair de France », comme le remarque un critique. Veuillot publie aussi de longs extraits du recueil dans L'Univers. Toutefois, il remarque que Hugo "insulte [s]a mère », tenancière, dans le vers "Ce Zoïle cagot naquit d'une Javotte » : Veuillot lui-même, dans sa recension du Rhin en 1842, s'était décrit comme un Zoïle. Veuillot trouve néanmoins une grande beauté aux Contemplations, publié en 1856, même s'il continue d'attaquer Hugo qui réclame le titre de proscrit. Il reconnaît aussi le chrétien Jean Valjean dans Les Misérables. Dans Les Odeurs de Paris, son livre de 1867, il fait l'éloge des Chansons des rues et des bois, où Hugo paraît enfin pousser à son achèvement la veine de Châtiments. Veuillot reconnaît qu'Hugo est « le plus grand poète du monde et peut-être de tous les temps ».

Pour sa part, Hugo chassé de Bruxelles en 1871 apprend que Veuillot l'a « appelé vieille citrouille »... Mais Veuillot a ajouté que ladite citrouille est « à moitié remplie de diamants », ce qu'Hugo sait aussi. L'inimitié des deux hommes, sans être la loyauté des adversaires, reste féconde : les Chansons des rues et des bois doivent aux Châtiments, qui doivent eux-mêmes à Veuillot. 


\section{Séminaire lié - The Stranger contre The Outsider : en lice pour le titre de L'Étranger}

Intervenante : Alice Kaplan, professeur de littérature française à Yale University.

\section{Séance 11 - Malices et vengeances de Sainte-Beuve}

\section{7 mars 2018}

Le maître-mot de Sainte-Beuve est « vengeance », suggère Wolf Lepenies. Il est, avec son Cahier vert et son Cahier brun, récemment publié, le peintre minutieux et souvent amer de la guerre littéraire de son époque. Sainte-Beuve constate ainsi que le temps est fini où la littérature pouvait être un simple exercice de l'esprit, confié à des auteurs affinés par leur mauvaise santé. Il faut à ce siècle « des athlètes à la puissante encolure » dont « la physiologie et l'hygiène » reflètent et caractérisent le talent.

Le monde littéraire décrit par Sainte-Beuve est fait d'ennemis et de rivaux. En choisissant ces derniers, on révèle l'esprit que l'on est. Les adversaires de SainteBeuve sont ses aînés - Guizot, Cousin, Villemain, Thiers -, ainsi qu'un certain nombre de critiques contemporains. Hugo n'en fait pas partie, parce qu'on ne fait pas la guerre publique à ses anciens amis, même et surtout lorsqu'ils sont devenus ses ennemis.

Sainte-Beuve ne manque pas de railler le charlatanisme de son époque: le charlatan se pousse du col et n'est pas si éloigné de l'athlète qui gonfle le sien. Le mal de l'époque tient à ce que ses grands auteurs ont cessé d'écrire pour leurs pairs au profit du peuple : c'est le cas d'Hugo et de George Sand. Chateaubriand n'échappe pas à la critique : c'est le type même de l'écrivain imposteur, auquel toute l'époque est complaisante. Le fait central de ce charlatanisme, c'est la rhétorique à laquelle s'adonne l'époque, et qui remplace la vraie bravoure. Sainte-Beuve est sévère envers ses contemporains qui ont délaissé la vraie guerre pour la guerre de plume. Mais il loue le parcours remarquable de Paul de Molènes : soldat et même bravache en critique, son amour de la guerre littéraire le mène à la carrière militaire, à rebours de son époque. Pour sa part, Baudelaire le vante ainsi : " "La guerre pour la guerre!" eût-il dit volontiers, comme d'autres disent "L'art pour l'art!" ”

Sainte-Beuve recourt souvent à la métaphore militaire pour parler de lui-même, ainsi quand il dit qu'il a «l'épée courte et fréquente», suggérant qu'il manie la plume comme une arme de poing. On ne peut écarter l'hypothèse d'une allusion sexuelle, Sainte-Beuve utilisant aussi la métaphore dans ce domaine lorsqu'il parle de tenir des troupes, d'assembler garnison. La métaphore vaut néanmoins surtout pour l'assemblage des idées et pour leur facilité de maniement à grande échelle par ces grands généraux que sont les écrivains de talent.

Sainte-Beuve regrette le temps des illustres batailles rangées, la guerre où l'on reçoit de rudes coups, et qui n'est pas la guérilla moderne. Il ne voit chez aucun de ses contemporains le respect qu'il faudrait avoir de l'adversaire, sans qui on n'est rien. Il se considère toujours lui-même comme le représentant d'une voie moyenne, y compris entre la modestie et la glorification, là où Hugo est constamment «baleine » ou « vessie ». Il récrit toute sa vie comme une suite de campagnes : il y a celle du Globe, puis celle de la Revue de Paris, celle de la Revue des Deux Mondes, celle de l'École normale supérieure, celle ratée du Collège de France, celle des Lundis... Ses armes, à chaque fois, sont les «malices et vengeances ». Comme Latouche, son modèle, Sainte-Beuve se plaît à glisser des couleuvres dans ses textes... qui lui font aussi ses couleurs. 


\title{
Séminaire lié - La guerre encore
}

Intervenante : Lydie Salvayre, écrivain.

\section{Séance 12 - Sainte-Beuve, Chateaubriand, Balzac : «Inde irae. »}

\author{
3 avril 2018
}

On trouve à la fin des Portraits de Sainte-Beuve, comme pour clore leur série, cet avis : «En critique, j'ai assez fait l'avocat, faisons maintenant le juge. » Il est de retour de la «campagne » de Liège et sur le point d'entamer celle des Lundis, dont la visée est claire: livrer enfin la «grande bataille rangée » qu'il prépare depuis longtemps contre la génération romantique avec laquelle il est prêt à rompre afin de révéler au grand jour l'esprit de vengeance qui anime en secret les relations de ses membres, et dont il fait la clef essentielle de la lecture des œuvres de son temps. Chez Rousseau, il trouve partout «l'arrière-pensée des représailles », et de même chez Vigny ou chez Latouche, roi de la couleuvre.

Les mémoires sont le genre le plus plein de vengeance, et Chateaubriand le plus exemplaire condottiere ou charlatan. Toute son œuvre, littéraire et politique, se comprend sur le modèle du Congrès de Vérone, texte de vengeance, ou plutôt de De Buonaparte et des Bourbons qui inaugure un ethos vengeur dont il est impossible de se défaire une fois qu'on l'a goûté. Selon Sainte-Beuve, les Furies, et non pas les Muses, ont présidé à l'entrée en politique de cet auteur.

Sainte-Beuve s'attarde sur la relation inamicale de Lamartine et Chateaubriand, qui s'entre-caressent en public. "Figure du faux grand homme », dit Lamartine de Chateaubriand à la messe. "Grand dadais », dit Chateaubriand de Lamartine, dans un propos rapporté par Sainte-Beuve, le jour où Lamartine est venu présenter chez Madame Récamier un livre à propos duquel Chateaubriand garde un silence obstiné. Ailleurs, il se plaît néanmoins à remarquer que Chateaubriand a certainement eu des raisons bien plus intéressées que celles qu'il a dites pour ne pas sauver la tête de son cousin Armand, condamné à mort, et dont Sainte-Beuve insinue qu'il ne fallait qu'un mot de Chateaubriand à Bonaparte pour le sauver. Le comble de la colère est sans doute atteint dans sa phrase malheureuse sur Decazes dont, dit-il, « les pieds lui ont glissé dans le sang », suggérant qu'il a peut-être trempé dans l'assassinat du duc de Berry. Sainte-Beuve se plaît à rappeler que la phrase n'est si forte - comme beaucoup de choses chez Chateaubriand - que parce qu'elle est celle d'un autre: on a effectivement suggéré à son auteur de la tronquer pour qu'il ne tombe pas trop dans son habituelle grandiloquence.

Mais c'est peut-être Balzac qui s'attire surtout la colère de Sainte-Beuve. L'inimitié des deux hommes remonte au roman Volupté de Sainte-Beuve, que Balzac a pourtant apprécié. Blessé par un article où Sainte-Beuve lui reproche d'être un inefficace combattant des lettres, Balzac décide de se venger en « refaisant Volupté » - Sainte-Beuve reviendra incessamment sur la formule - sous la forme de son Lys dans la vallée. Sainte-Beuve décrira régulièrement Balzac comme un «marchand à la toilette », lui reprochant ses romans écrits pour les femmes, chiffons perdus au milieu des taches de graisse. Sainte-Beuve trouve que Balzac est un mauvais stratège littéraire et un génie brouillon, qui a besoin de nombreux coups d'essais avant de produire une œuvre bonne, comme un général gaspillant indignement le sang de ses soldats. 
Balzac répond à Sainte-Beuve que ses attaques sont celles d'un couard et d'un impuissant, et remarque qu'il a mis bien plus de temps à faire Volupté que lui Le Lys dans la vallée. Sainte-Beuve continue de répondre à Balzac jusque dans l'article nécrologique qu'il écrit en 1850. Revenant incessamment à cette scène première de Volupté, il explique toute l'attitude de Balzac par le besoin de la vengeance : "De là sa colère, son besoin de vengeance, et son intrusion sur les terres de Port-Royal. »

Le $\mathrm{XX}^{\mathrm{e}}$ siècle ne connaît pas de grande rivalité comme celle de Veuillot et de Hugo, ou comme celle de Sainte-Beuve et de Balzac. Est-ce parce que les guerres ont apaisé la vie littéraire ? Est-ce parce que l'État a enfin conquis le monopole de la violence légitime ? Est-ce parce que la littérature elle-même est moins vivace ?

\section{Séminaire lié - Conversation}

Intervenant : Christophe Boltanski, artiste plasticien.

\section{COLLOQUE - LA LITTÉRATURE COMME LIEU DU NON-CONFLIT (II)}

Dans le prolongement du cours et du séminaire d'Antoine Compagnon, et comme pour lui faire pendant, la seconde édition de ce colloque fermé, coorganisé par Antoine Compagnon et Odile Bombarde le 13 avril 2018 et accueilli par la fondation Hugot du Collège de France, s'est construite comme une réflexion sur le rapport de la littérature et des formes de la pacification.

- Jean-Baptiste Amadieu : «L'admiration littéraire du censeur pour le censuré »;

- Céline Surprenant : «Sur une illusion du sens commun»;

- André Guyaux : «La conversion comme entreprise pacificatrice. Huysmans, Verlaine et compagnie »;

- Odile Bombarde : « La correspondance comme locus amoenus : Yves BonnefoyGeorges Henein »;

- Jacqueline Lichtenstein : "Querelles autour d'un tableau : Eliezer et Rebecca de Poussin »;

- Rémi Labrusse : " "Préhistoire" : un mot pour guérir de la nuit du temps ?».

\section{Colloque - HISTOIRE LITTÉRAIRE : NOUVEAUX OBJETS, NOUVELLES MÉTHODES}

Le colloque du printemps dernier avait réuni les auteurs de dix livres touchant à la théorie de la littérature et publiés depuis 2007, en retenant un livre pour chacune des années de la décennie. Pour cette édition du 31 mai 2017, le renouveau de l'histoire littéraire est au programme, par l'intermédiaire d'une dizaine d'ouvrages récents, mais affranchis cette fois de la contrainte du livre par an, afin que toutes les époques puissent être représentées. L'ambition reste la même : illustrer la recherche actuelle sur la littérature française, montrer sa variété et sa robustesse, rendre hommage à son originalité.

- Antoine Compagnon (Collège de France) : Ouverture ;

- Olivier Guerrier (Toulouse II) : «Montaigne et le "moment Renaissance" », à propos de Rencontre et reconnaissance: les Essais ou le jeu du hasard et de la vérité (2016) ;

- Anne Régent-Susini (Paris III) : «Une expérience des limites ? Rhétorique, religion et littérature » à propos de Bossuet et la rhétorique de l'autorité (2011) ; 
- Sophie Lefay (Orléans): «La littérature hors les livres», à propos de L'Éloquence des pierres. Usages littéraires de l'inscription au XVIII siècle (2015);

- Jean-Baptiste Amadieu (CNRS) : «Des archives au discours savant », à propos de La Littérature française du XIX e siècle mise à l'Index. Les Procédures (2017);

- Sophie Basch (Paris IV) : «De la brocante considérée comme un des beauxarts », à propos de Rastaquarium. Marcel Proust et le «modern style». Arts décoratifs et politique dans À la recherche du temps perdu (2014);

- Jean-Pierre Bertrand (Liège) : «Réflexion faite», à propos de Inventer en littérature. Du poème en prose à l'écriture automatique (2015);

- Bertrand Westphal (Limoges) : «La littérature à l'échelle planétaire », à propos de La Cage des méridiens. La littérature et l'art contemporain face à la globalisation (2016) ;

- Vincent Kaufmann (Saint-Gall) : «Après les avant-gardes, le spectacle», à propos de Dernières Nouvelles du spectacle. Ce que les médias font à la littérature (2017);

- Hélène Merlin-Kajman (Paris III) : «Le traumatique : quelques précisions », à propos de Lire dans la gueule du loup. Essai sur une zone à défendre, la littérature (2016) ;

- Catherine Coquio (Paris VII) : «Le témoignage : critique de la mémoire et critique des œuvres », à propos du Mal de vérité ou l'utopie de la mémoire (2015).

\section{PROGRAMme «PASSAGE DES DISCIPLINES »}

\section{Journée d'études - Les professeurs du Collège de France et de l'ESPCI}

Cette journée d'études, qui s'est tenue le 14 novembre 2017, a été organisée par Antoine Compagnon et Céline Surprenant, en collaboration avec Catherine Kounelis, dans le cadre du programme « Passage des disciplines », et avec le soutien de PSL Research University. Il s'est agi de questionner l'histoire de la constitution des disciplines scientifiques, en étudiant la répartition des chaires créées entre le Collège de France et l'ESPCI, et notamment l'histoire des chevauchements entre les deux établissements, auxquels des physiciens ou des chimistes ont successivement ou simultanément appartenu.

- Antoine Compagnon (Collège de France) et Catherine Kounelis (Bibliothèque et Centre de ressources historiques de l'ESPCI) : Introduction;

- Gérard Emptoz (université de Nantes) : «Paul Schützenberger (1829-1897), un parcours remarquable entre sciences et techniques »;

- Danielle Fauque (université Paris-Sud): «Charles Dufraisse (1885-1969), L'effet "anti-oxygène". Du laboratoire du Collège de France à la croisée des réseaux issus de la Grande Guerre »;

- Martha-Cecilia Bustamante (université Paris-Diderot) : «Les Difficultés de la théorie du rayonnement ou une conception pédagogique nouvelle : Paul Langevin au Collège de France au début du XX $\mathrm{X}^{\mathrm{e}}$ siècle »;

- Éric Brian (EHESS) : «Physique et chimie industrielle dans l'Encyclopédie française : Lucien Febvre et la Nouvelle histoire sous le rayonnement de l'ESPCI ». 


\section{Colloque - Einstein au Collège de France}

Ce colloque, organisé par Antoine Compagnon et Céline Surprenant les 11 et 12 juin 2018, avec le soutien de la fondation Hugot du Collège de France et de PSL Research University, fait partie du programme «Passage des disciplines ». Avec pour fil conducteur la visite d'Einstein au Collège en 1922, suite aux efforts du physicien Paul Langevin pour le faire venir, le colloque s'est intéressé à l'impact des idées d'Einstein sur la physique française et, plus largement, dans la formation des savoirs et des arts, à partir des années 1910 en France et au-delà.

- Antoine Compagnon (Collège de France), Jean Dalibard (Collège de France), Jean-François Joanny (ESPCI) : Ouverture.

\section{Avant Einstein - La physique au Collège}

- Françoise Balibar (université Paris-Diderot) : «Einstein perçait-il déjà sous Mascart?».

\section{La physique d'Einstein}

- Françoise Combes (Collège de France) : «La constante cosmologique, la plus grande erreur d'Einstein »;

- Jean-François Joanny (ESPCI) : «Paul Langevin et le mouvement brownien »;

- Bernard Derrida (Collège de France) : «Einstein et les fluctuations ».

\section{Einstein à Paris, Paul Langevin et Henri Bergson}

- Élie During (université Paris-Nanterre) : «Une étrange obstination : Bergson, Langevin, et le temps des jumeaux »;

- Thibault Damour (IHES) : «Comment penser le temps après Einstein ? »;

- Jimena Canales (University of Illinois, Urbana-Champaign) : «After-effects of Einstein's Paris visit».

\section{Einstein à Princeton}

- Jürgen Renn (Max Planck Institute for the History of Science) et Hanoch Gutfreund (Hebrew University of Jerusalem) : « The Formative Years of Relativity ».

\section{Einstein, Poincaré et Frédéric Joliot-Curie}

- Peter Galison (Harvard University) ; « Einstein and Poincaré » ;

- Michel Paty (CNRS, Laboratoire Sphere) : «Einstein en voyage et les nouvelles perspectives de la physique dans les années 1920 et $1930 »$;

- Hélène Langevin-Joliot (CNRS, Institut de physique nucléaire d'Orsay): « Témoignage ».

- Pierre Joliot (Collège de France), «Frédéric Joliot : une certaine idée de la recherche ».

\section{Einstein et la culture moderne}

- Serge Haroche (Collège de France) : «Einstein et les quanta»;

- William Marx (université Paris Nanterre) : «Valéry et Einstein, ou le poème de la relativité »;

- Claudine Tiercelin (Collège de France) : «Relativité et relativisme»; 
- Éric Brian (EHESS) : «Einstein au bord de la falaise. Les attentes des historiens parisiens après ses conférences au Collège de France (1922-1943) »;

- Jean-Michel Rabaté (University of Pennsylvania) : «Einstein, Russell, Whitehead et le paradigme moderniste anglo-saxon en $1922 »$;

- Antoine Compagnon (Collège de France) : Conclusion.

\section{JoURNÉES D'ÉTUdES - «PEINT D'APRÈS NATURE ». L'ART DU PORTRAIT EN EUROPE ENTRE CONTRE-RÉFORME ET BAROQUE (1563-1623)}

Ces trois journées d'études (19-21 mars 2018), organisées par Francesco Solinas, ont eu pour ambition de questionner la naissance du portrait réaliste en Europe autour de la Contre-Réforme, en replaçant notamment le genre dans un certain nombre de logiques économiques et symboliques.

- Francesco Solinas (Collège de France, République des savoirs) : Bienvenue ;

- Federica Veratelli (université de Parme): «Le portrait du marchand entre construction identitaire et gestion de la réputation »;

- Charles Dominique Fuchs (musée Stibbert, Florence) : «Portraits en bronze de la Renaissance à la Contre-Réforme. Matière et pouvoir »;

- Alexandra Zvereva (centre Roland Mousnier, CNRS, université Paris-Sorbonne) : " "C'est moi que je peins" : l'évolution du portrait aulique en France au temps des guerres de religion »;

- Claudio Strinati (surintendant honoraire du Polo Museale Romano) : «Le genre du portrait à la cour de Rome entre Scipione Pulzone (1544-1598) et le Caravage (1571-1610)»;

- Kelley Helmstutler Di Dio (université du Vermont) : «Sculpted portraits of the Spanish Kings, 1549-1640» ;

- Cecilia Mazzetti Di Pietralata (université de Chieti) : «Portraits des Habsbourg et portraits de famille entre Vienne, Madrid et Rome (XVI $-\mathrm{XVII}^{\mathrm{e}}$ siècles) »;

- Elizabeth Oy-Marra (université de Mainz) : «Quelle vérité dans le portrait ? Similitude et imitation dans le discours théorique chez Ludovico Castelvetro (15051571)»;

- Emanuele Pellegrini (IMT, School for advanced studies, Lucques) : «Lieux et genres du portrait : du roi François ${ }^{\mathrm{er}}$ au prince Léopold de Médicis»;

- Ilaria Andreoli (Item, CNRS, Fondazione Giorgio Cini, Venise) : «Portraits bridés. Visages japonais en Italie entre $\mathrm{XVI}^{\mathrm{e}}$ et $\mathrm{XVII}^{\mathrm{e}}$ siècle»;

- Luciano Arcangeli (directeur honoraire de la Soprintendenza delle Marche) : «Federico Barocci (1535-1612) et le portrait "empathique" »;

- Heiko Damm (université de Mainz) : «Les portraits des artistes de Bartolomeo Passarotti (1529-1592)»;

- Blaise Ducos (musée du Louvre, Paris) : «Mantoue et Prague, la rivalité entre Frans Pourbus le Jeune (1569-1622) et Hans von Aachen (1552-1615)» ;

- Peter M. Lukehart (Casva, Center for advanced study in the visual arts, Washington): «Le sang d'un peintre: les portraits allégoriques de Giovanni Battista Paggi (1554-1627) »;

- Sebastian Schütze (université de Vienne) : «Changing paradigms: the poetics of portraiture from Aretino to Giovan Battista Marino »;

- Laura Bartoni (Uninettuno, université télématique internationale) : «Représentation et fonctions du portrait dans les collections des princes Colonna »; 
- Gilles Sauron (université Paris-Sorbonne) : «L'Antiquité reconstituée à la Renaissance : le Pompée Spada »;

- Lucia Calzona (Galleria Borghese, Rome) : «L'image de la femme entre Contre-Réforme et Baroque. Notes d'une recherche en cours »;

- Sybille Ebert-Schifferer (Bibliotheca Hertziana, Max-Planck-Institut für Kunstgeschichte) : " "Con arte, ma senza similitudine". Le problème de Caravage peintre de portraits $»$;

- Roberto Contini (Gemäldegalerie, Staatliche Museen zu Berlin) : «Une idée pour Caravage peintre de portraits »;

- Élodie Vaysse (Musée national des châteaux de Malmaison et Bois-Préau): «Éloge de l'accessoire ? Un état du portrait à Paris au début du XVII ${ }^{\mathrm{e}}$ siècle ».

\section{ENSEIGNEMENTS À L'ÉTRANGER}

Université Diego Portales, Santiago. Institut français du Chili, Santiago. Institut français, Buenos Aires. Université de Buenos Aires. Université catholique de Buenos Aires. Université de Zürich. Société académique de Genève. Institut français, Stuttgart. Institut national genevois, Genève. Institut français, Beyrouth. Université Saint-Joseph, Beyrouth.

\section{RECHERCHE}

\section{ACTIVITÉS DE L'ÉQUIPE}

Francesco Solinas, maître de conférences, a consacré un travail important à la conception et l'organisation des journées internationales d'études sur le «Peint d'après nature ». L'art du portrait en Europe entre Contre-Réforme et Baroque (1563-1623), qui se sont tenues au Collège de France en mars 2018, et dont la parution des actes est prévue pour 2019. En collaboration avec Pierre Caye (CNRS), il a également participé à la rédaction et à l'édition du troisième volume des Cahiers de l'Ornement, actes du séminaire Ornement (Collège de France, CNRS, 20132015), et dont la parution est prévue pour la fin de l'année 2018 aux éditions De Luca à Rome. Il a enfin participé à la rédaction et à l'édition des Lettres de Léonard de Vinci aux puissants de son temps, sous la direction de Pietro Cesare Marani (Polytechnique de Milan), premier volume de la série Lettres d'artistes et lettres sur l'art relative au séminaire conçu et organisé par Francesco Solinas au Collège de France (CNRS, Labex Transfers, 2017), et dont la parution est prévue pour la fin 2018 aux mêmes éditions De Luca.

Corinne Maisant, maître de conférences, a poursuivi la constitution d'une base de données d'iconographie documentaire centrée sur les tableaux et dessins illustrant des scènes du répertoire théâtral, en se concentrant sur celles des $\mathrm{XVII}^{\mathrm{e}}$ et XVIII ${ }^{\mathrm{e}}$ siècles (Coypel, de Troy, Van Loo, etc.).

Odile Bombarde, maître de conférences, a organisé le colloque de l'Institut d'études littéraires à la fondation Hugot du Collège de France: «La littérature comme lieu du non conflit, 2 », le 13 avril 2018 ; elle y a proposé la communication suivante: La correspondance comme locus amœnus: Yves Bonnefoy-Georges Henein. Elle a également élaboré la journée «La Correspondance d'Yves 
Bonnefoy » au Collège de France, le 22 juin 2018, journée qui comportait un débat avec les intervenants.

Céline Surprenant, chercheur associée, a pris en charge une grande partie des activités du programme «Passage des disciplines », soutenu par PSL Research University de 2017 à 2020. Elle a notamment travaillé à la mise en place du colloque «Einstein au Collège de France », coorganisé par Antoine Compagnon, Jean Dalibard et Jean-François Joanny, et à celle de la journée d'études sur «Les professeurs du Collège de France et de l'ESPCI », organisée en collaboration avec Catherine Kounelis de la bibliothèque et du centre de ressources historiques de l'ESPCI.

Lucile Peycéré, archiviste du programme «Passage des disciplines », a poursuivi ses recherches de «rapports de présentation» aux Archives nationales, afin de compléter la collection numérisée des rapports sur les disciplines littéraires et scientifiques rédigés par les professeurs au moment de la sélection des chaires.

Clément Girardi, ATER, a consacré l'essentiel de son travail de recherche à la rédaction de sa thèse de doctorat intitulée Le Morceau de sucre et la fleur de papier. Écrire avec et contre Bergson (1890-1940), conduite à l'université de la Sorbonne sous la direction de M. Didier Alexandre, et soutenue le 21 juin 2018 devant M. Antoine Compagnon, Bruno Clément, Michel Murat et Frédéric Worms. Il a également pris en charge la préparation d'un volume des Annales bergsoniennes, à paraître en 2019 sous sa direction, et consacré à la question du rapport des écrivains à la philosophie de Bergson, tout au long du $\mathrm{XX}^{\mathrm{e}}$ siècle et à l'échelle de plusieurs aires linguistiques. À paraître, un compte rendu du livre d'Arnaud François sur $L a$ Philosophie d'Émile Zola. «Faire de la vie » (2017), dans la revue Esprit.

Matthieu Vernet s'est consacré cette année à la transcription diplomatique, à l'annotation et à la publication du Cahier 7 des manuscrits d'À la recherche du temps perdu. Il a commencé, en outre, son travail d'édition des cahiers primitifs de La Recherche, en préparant avec Antoine Compagnon un nouveau scénario pour le projet inachevé par Proust de Contre Sainte-Beuve.

Alexandre de Vitry, enseignant au collège Pierre-Curie de Goussainville (Vald'Oise) et chercheur associé à la chaire d'Antoine Compagnon, a travaillé à concevoir un projet d'exploitation des archives d'Augustin Cochin (1876-1916), en vue d'un classement du fonds et d'une reprise intégrale de l'édition des œuvres de ce dernier. Le projet a été soutenu par le prix Jeune chercheur 2018 de la fondation des Treilles. Il a par ailleurs fait la collecte des différentes communications du colloque « Histoire et historiens des idées » qu'il avait organisé avec David Simonetta au Collège de France, du 18 au 20 mai 2016, sous le patronage des chaires d'Antoine Compagnon et d'Alain de Libera, en vue d'une publication. Il a enfin assisté Antoine Compagnon dans la collecte et la reprise de différentes participations à son séminaire et à son colloque de 2016 sur les Chiffonniers littéraires, en vue de la parution fin 2018 d'un numéro spécial de la Revue d'histoire littéraire de la France. À paraître : «Baudelaire et ses frères », en cours d'examen par le comité de lecture de la $R H L F$, et «Le "fin langage français" de Péguy: style national ou stylistique nationaliste?», communication prononcée le 29 juin 2018 dans le cadre du colloque international « Du style des idées: nationalisme et littérature (1870-1920)», organisé par Stéphanie Bertrand et Sylvie Freyermuth à l'université du Luxembourg.

La chaire Littérature française moderne et contemporaine d'Antoine Compagnon constitue l'essentiel de l'équipe Respublica Literaria de la République des Savoirs, USR 3608 du CNRS. 


\section{AUTRES RESPONSABILITÉS SCIENTIFIQUES}

Directeur de l'USR 3608 «République des Savoirs: littérature, sciences, philosophie ». Membre du conseil scientifique du Collegium de Lyon. Membre du conseil d'administration de la fondation Singer-Polignac. Membre du conseil d'administration de la Bibliothèque nationale de France. Président du conseil scientifique de la Bibliothèque nationale de France.

Thèse soutenue sous la direction du professeur : Martine Lavaud, «Un siècle de révolution industrielle et médiatique, 1830-1940. Représentations, luttes et adaptations de l'individu littéraire », HDR, Sorbonne Université, juin 2018.

\section{PuBLiCATIONS}

BOMBARDE O., édition établie, introduite et annotée avec P. LABARTHE de : BONNEFOY Y., Correspondance, tome I, Paris, Les Belles Lettres, 2018.

BOMBARDE O., «La forge du père : "Jamais je n'avais franchi ces lourdes grilles" », Europe, $\mathrm{n}^{\mathrm{O}} 1067,2018$, p. 202-215.

Compagnon A., Les Chiffonniers de Paris, Paris, Gallimard, coll. « Bibliothèque illustrée des histoires », 2017, prix Guizot de l'Académie française.

Compagnon A., Baudelaire devant l'innombrable, édition revue et corrigée, augmentée d'une postface de l'auteur, Paris, Presses universitaires de Paris-Sorbonne, 2018.

Compagnon A. (dir.), Dix ans de théorie, Acta Fabula, vol. 19, nº 1, 2018.

Compagnon A., «Bac sélection université », Le Débat, n 199, 2018.

GIRARDI C., «Une mathématique pour Bergson: figures de la science suarésienne », in M. Murat (dir.), La Couronne littéraire d'André Suarès, Paris, Classiques Garnier, 2017.

GIRARDI C., "Creative criticism vs. Creative evolution: Thibaudet's experiences in Bergsonian historiography », in M. SOMERS et B. LAMBRECHT (dir.), Writing Literary History: 1900-1950, Louvain, Peeters Publishers, 2018, p. 25-38.

Vernet M., «Le Massif Central de la critique française : 1900-1930», in J.-L. Diaz et V. Feuillebois (dir.), Lectures critiques du romantisme aи XXe siècle, Paris, Classiques Garnier, 2018, p. 17-27.

VERNET M., «Baudelaire est moderne là où on ne l'attend pas », propos recueillis par B. Hernandez, Le Point, 7 juillet 2017.

Vernet M., «Du côté de chez Proust », Le Point : "Charles Baudelaire. J'ai pétri de la boue et j'en ai fait de l'or », no 23, juillet-août 2017, p. 89-91.

VITRY A. de, «Péguy philosophe », Commentaire, $\mathrm{n}^{\circ}$ 160, vol. 4, 2017, p. 938-940 : compte rendu de RiQuier C., Philosophie de Péguy ou les mémoires d'un imbécile, Paris, PUF, 2017.

VITRY A. de et BRULEY P., «Aperçu bibliographique : [bibliographie sur Charles Péguy: octobre 2016-octobre 2017; complément 2001-2016]», Bulletin de l'Amitié Charles Péguy, $\mathrm{n}^{\mathrm{o}} 160,2017$, p. 407-420.

VitRY A. de, «Le lettré 1900: une figure individualiste?», in A. MinZETANu et W. MARX (dir.), Le Lettré : définitions et enjeux. Actes du colloque organisé à l'université Paris Nanterre le 27 janvier 2017, Revue Silène, Centre de recherches en littérature et poétique comparées de Paris Ouest-Nanterre-La Défense, 2017. 
VITRY A. de, « Péguy en Barbarie », Philitt. Revue de philosophie et de littérature, $\mathrm{n}^{\circ}$ 5, 2017, p. 8 .

VITRY A. de, « Péguy es-tu là ? », Le Débat, Paris, Gallimard, nº 199, 2018, p. 115-124, DOI : 10.3917/deba.199.0115.

VITRY A. de, «Port-Royal aux Cahiers de la Quinzaine », in S. ICARD, L. PlazenET et G. MÉTAyer (dir.), Port-Royal et la République : 1940-1629, Paris, Société des Amis de PortRoyal, coll. «Chroniques de Port-Royal », nº 68, 2018, p. 85-102. 